\title{
miR-127-5p promotes chondrogenic differentiation in rat bone marrow mesenchymal stem cells
}

\author{
ZHAOLONG XUE $^{1}$, YANLI MENG ${ }^{1}$ and JIANHUA GE ${ }^{2}$ \\ ${ }^{1}$ Department of Orthopedics, Shanghai Jiading Nanxiang Hospital, Shanghai; ${ }^{2}$ Department of Bone and Joint Surgery, \\ The Affiliated Hospital of Southwest Medical University, Luzhou, Sichuan, P.R. China
}

Received January 10, 2017; Accepted June 20, 2017

DOI: $10.3892 /$ etm.2017.4711

\begin{abstract}
The effect and related mechanisms of miR-127-5p on the cartilage differentiation of rat bone marrow mesenchymal stem cells (BMSCs) was investigated. Rat BMSCs were generated and transfected with miR-127-5p, RT-PCR and Safranin O staining were used to detect the effect of miR-127-5p on the cartilage differentiation of rat BMSCs. Western blot analysis was used to detect the related mechanisms of miR-127-5p on the cartilage differentiation of rat BMSCs. Genes related to cartilage differentiation such as Sox9, collagen II and aggrecan were significantly increased in the group which were transfected with miR-127-5p, while collagen $\mathrm{X}$, which was related to cartilage hypertrophy, was decreased in the miR-127-5p transfected group. Safranin O staining revealed that the expression of chondroitin sulfate was significantly increased in the group of miR-127-5p, than the miRNA control group. Western blot analysis showed that miR-127-5p transfection promoted the expression of Sox9, while decreased the expression of Runx2 of rat BMSCs. In conclusion, via increasing the expression of Sox 9 and decreasing the expression of Runx 2, miR-127-5p could promote cartilage differentiation and decrease cartilage hypertrophy of rat BMSCs.
\end{abstract}

\section{Introduction}

The regeneration ability of articular cartilage is limited, and tiny trauma can induce severe pain and dysfunction of articular. A good treatment strategy for osteoarthritis (OA) is to in vitro build cartilage tissues, which have the same biological features as articular cartilage, thus restoring the defects of cartilage (1). Currently, autologous cartilage transplantation is the most common way to restore the defects of articular cartilage. Autologous cartilage transplantation means human articular

Correspondence to: Dr Jianhua Ge, Department of Bone and Joint Surgery, The Affiliated Hospital of Southwest Medical University, 25 Taiping Street, Luzhou, Sichuan 646000, P.R. China

E-mail: ehiobl@163.com

Key words: miR-127-5p, bone marrow mesenchymal stem cells, cartilage differentiation, hypertrophy, Sox 9 cartilage cells are generated from human body, cultured and amplified in vitro, and eventually the amplified cartilage cells were transplanted to the defected articular cartilage. However, dedifferentiation is common with in vitro cultured articular cartilage, meaning that the expression of collagen II and aggrecan representative of hyaline cartilage of extracellular matrix are decreased, while collagen $\mathrm{X}$ representative of cartilage hypertrophy is upregulated. Therefore, cartilage tissues built in autologous cartilage transplantation usually are not hyaline cartilage, and the repairing effect of articular cartilage defects are quite limited (2).

Another replacement of autologous cartilage transplantation is to build cartilage tissues by stem cells or biological materials, and bone marrow mesenchymal stem cell (BMSC) is a very attractive cell (3). To fulfill this purpose, it is necessary to fully understand the molecular mechanism of the differentiation of BMSCs into chondrocytes.

miRNAs are small, single strand RNA molecules composed of 18-25 nucleotides. Combined with related proteins to form RNA-induced silencing complex (RISC), miRNAs could take part in post-transcription regulation of target genes by inhibiting translation or promoting degradation of target genes. Accumulating evidence has shown that many miRNAs are related with the initiation, pathologic grade, clinical stages, chemotherapy resistance and prognosis of tumors and even directly take part in the initiation and progression of neoplasms including leukemia,lymphoma, lung and colon cancer.Indifferent tumors, miRNAs displayed particular expression patterns and have potential of differentiating tumors types and early diagnosis (4). miR-127, a small, non-coding RNA with overlapped gene structure, plays a role in lung development, embryogenesis and tumor initiation (5). Previous studies revealed that miR-127 showed downregulation in various tumors including malignant gliomas, gastric and liver cancer (6-8). It is reported that inhibition of miR-127 expression promoted the initiation of liver cancer (9) and was closely linked to the oncogenesis of diffuse large B-cell lymphoma (10). Also, miR-127 could activate JNK signaling pathway and promote lung inflammatory injury (11). In particular, researchers indicated that miR-127-5p were downregulated in OA cartilage tissues compared with normal tissues and it promoted the development and progression of OA by regulating the expression of MMP-13 in cartilage cells (12). From all the above, we hypothesized that miR-127-5p may play a critical role in the differentiation of the cartilage. 
Until now, the roles of miR-127-5p in regulating rat BMSC differentiation into cartilage remain unclear. In this study, we investigated the effect and related mechanisms of miR-127-5p on the cartilage differentiation of rat BMSCs to provide theoretical basis and experimental evidence for construction of hyaline cartilage with stable phenotypes.

\section{Materials and methods}

Materials. The following were obtained: DMEM media, F12 media, collagen I, trypsin and fetal bovine serum (FBS) were from Gibco Life Technologies (Carlsbad, CA, USA). miRNAs analogues were from Guangzhou Ruibo Biological Technology Co., Ltd. (Guangzhou, China). Lipofectamine ${ }^{\mathrm{TM}}$ RNAiMAX was from Invitrogen Life Technologies (Carlsbad, CA, USA). Primary rabbit polyclonal Sox 9 antibody (dilution, 1:1,000; cat. no. sc-20095) and rabbit polyclonal Runx2 antibody (dilution, 1:1,000; cat. no. sc-10758) were from Santa Cruz Biotechnology, Inc. (Santa Cruz, CA, USA) and horseradish peroxidase coupled secondary antibodies were also from Santa Cruz Biotechnology, Inc.. PE labeled anti-CD34 (dilution, 1:100; cat. no. 343604), CD44 (dilution, 1:100; cat. no. 103007), CD45 (dilution, 1:100; cat. no. 304016), CD90.2 (dilution, 1:100; cat. no. 105314) FACS and comparisons were from BioLegend, Inc. (San Diego, CA, USA). ECL kit was from Beyotime Institute of Biotechnology (Shanghai, China), TRIzol Reagent was from Invitrogen Life Technologies, RT-PCR kit, Taq polymerases and dNTPs were from MBI Fermentas, Inc. (Burlington, ON, Canada) and IQ SYBR Green Supermix was from Bio-Rad Laboratories, Inc. (Hercules, CA, USA). Cartilage induction solution was from Saiye Health Research Center (Taicang) Co., Ltd., (China). Safranin O staining was from Boster Bio-Engineering (Wuhan, China) and Protein Quantity Assay kit was from Boster Bio-Engineering.

Isolation and culture of BMSCs. Sprague-Dawley rats at 12 weeks were purchased from Shanghai Laboratory Animal Center (SLAC), CAS, (Shanghai, China) and all the animal experiments were approved by the Ethics Committee of Nanxiang Hospital. After sacrificing the rats under anaesthesia, we isolated bilateral limbs, cut off metaphysis and flushed out medulla with syringe under sterile conditions. Single cell suspension was prepared with PBS and centrifuged at $1,000 \mathrm{x}$ g for $10 \mathrm{~min}$. We discarded the supernatant and suspended cells with DMEM/F12 media. Suspended cells labeled as primary cells were plated in $25 \mathrm{~cm}^{2}$ culture bottle and cultured in incubators with $37^{\circ} \mathrm{C}$ and $5 \% \mathrm{CO}_{2}$. We changed the medium every 2 days and observed the growth and morphology under inverted microscope (BX-42; Olympus, Tokyo, Japan). Cells were passaged with $0.25 \%$ trypsin at $1: 2$ when they almost merged.

Differentiation of CDs on the surface of rat BMSCs with flow cytometry. Rat BMSCs at passage 3 were trypsinized for 2 min at $25^{\circ} \mathrm{C}$ and added low-glucose DMEM with $10 \%$ FBS to stop digestion. Then cells were suspended with medium at a density of $5 \times 10^{5} / \mathrm{ml}$ after being centrifuged at $750 \times \mathrm{g}$ for $5 \mathrm{~min}$. PE/Cy7-conjugated anti-CD34 antibodies $(10 \mu \mathrm{l})$, PE-conjugated anti-CD45 antibodies $(6 \mu \mathrm{l})$, PE-conjugated
anti-CD44 antibodies (6 $\mu 1)$, PE-conjugated anti-CD90.2 antibodies $(6 \mu \mathrm{l})$ and their isotype control antibodies were added to $100 \mu \mathrm{l}$ cell suspensions, respectively. They were incubated at $4^{\circ} \mathrm{C}$ for $30 \mathrm{~min}$ and on the shaker at $37^{\circ} \mathrm{C}$ for $60 \mathrm{~min}$, then cells were washed twice with PBS and suspended with $200 \mu$ l PBS for flow cytometry detection.

Alcian blue staining of BMSCs induced chondroblasts microgroups. Cell groups (induced for 0, 7 and 17 days, respectively) were fixed in $4 \%$ paraformaldehyde for $20 \mathrm{~min}$ and washed for three times with $0.01 \mathrm{M}$ PBS (5 min each time). The washed cells were dehydrated with graded ethanol (30\%-50\%70\%-80\%-90\%-100\%), vitrificated by dimethylbenzene and paraffin-embedded. The cell groups went through dewaxing and dehydration before being sliced for $5 \mu \mathrm{m}$. Then they were stained with Alcian blue (pH 2.5) for $30 \mathrm{~min}$, washed with running water for $5 \mathrm{~min}$ and dehydrated with graded ethanol, vitrificated by dimethylbenzene, then the pieces were sealed by neutral gum.

Collagen II immunohistochemistry staining of BMSCs induced chondroblasts microgroups. Chondroblasts (induced for 0,7 and 17 days, respectively) were fixed, dehydrated and embedded. BMSCs which were not induced were considered as control group. SABC-DAB color assay was used to detect collagen II expression.

miR-127-5p transfection in BMSCs. BMSCs at passage 3 were plated in 6-well plates and transfected with Lipofectamine ${ }^{\mathrm{TM}}$ RNAiMAX when cells reached a density of $50-60 \%$. Cells were transfected with miR-127-5p analogues (300 pmol) for the experimental group and meaningless sequence (300 pmol) for the negative control group. Thirty-six hours after transfection, BMSCs were induced to differentiate into chondroblasts and constructed of cartilage micro-clusters by centrifugation.

Chondroblasts differentiation induced by BMSCs and construction of cartilage micro-clusters. BMSCs in both experimental group and control group were induced to differentiate. The cartilage induction solution was composed of low-glucose DMEM medium, L-proline (40 mg/l), indomethacin $(1 \%)$, sodium pyruvate $(100 \mathrm{mg} / \mathrm{l})$, dexamethasone $(100 \mathrm{nmol} / \mathrm{l})$, FBS (1\%) and ascorbic acid $(50 \mathrm{mg} / \mathrm{l})$. BMSCs at 1 week post-induction were digested, suspended and centrifuged. The cartilage induction solution was added shortly after supernatant was abandoned. Cells were cultured in incubators with $5 \% \mathrm{CO}_{2}$ at $37^{\circ} \mathrm{C}$ and changed medium every 4 days. The formed cartilage was sliced 2 weeks later.

RT-PCR assay. BMSCs at 1 week post-induction were lysed with TRIzol reagent and extracted RNA. After the whole RNA extraction, a PrimeScript RT kit (Takara Bio, Dalian, China) was used for reverse transcription. The reversed cDNA were ready for RT-PCR. Primers were labeled with SYBR-Green I. The primers were as follows: Sox9 (forward: 5'-AAAGGAAG GAAGGGAAGAAAGG-3', reverse: 5'-AATATGGCATCTT TCGATTTCTG-3'); collagen II (forward: 5'-CAAGTCGCTG AACAACCAGA-3', reverse: 5'-GCCCTCATCTCCACATC ATT-3'); aggrecan (forward: 5'-ACGGTGGGAAATGAAAG 

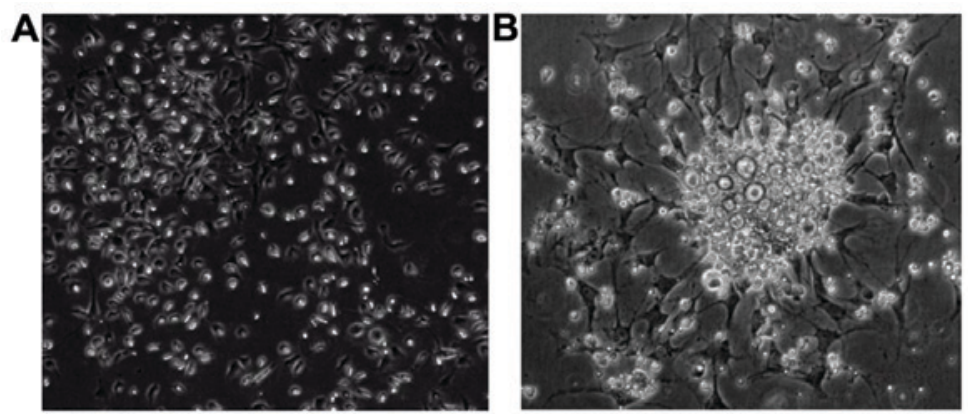

E
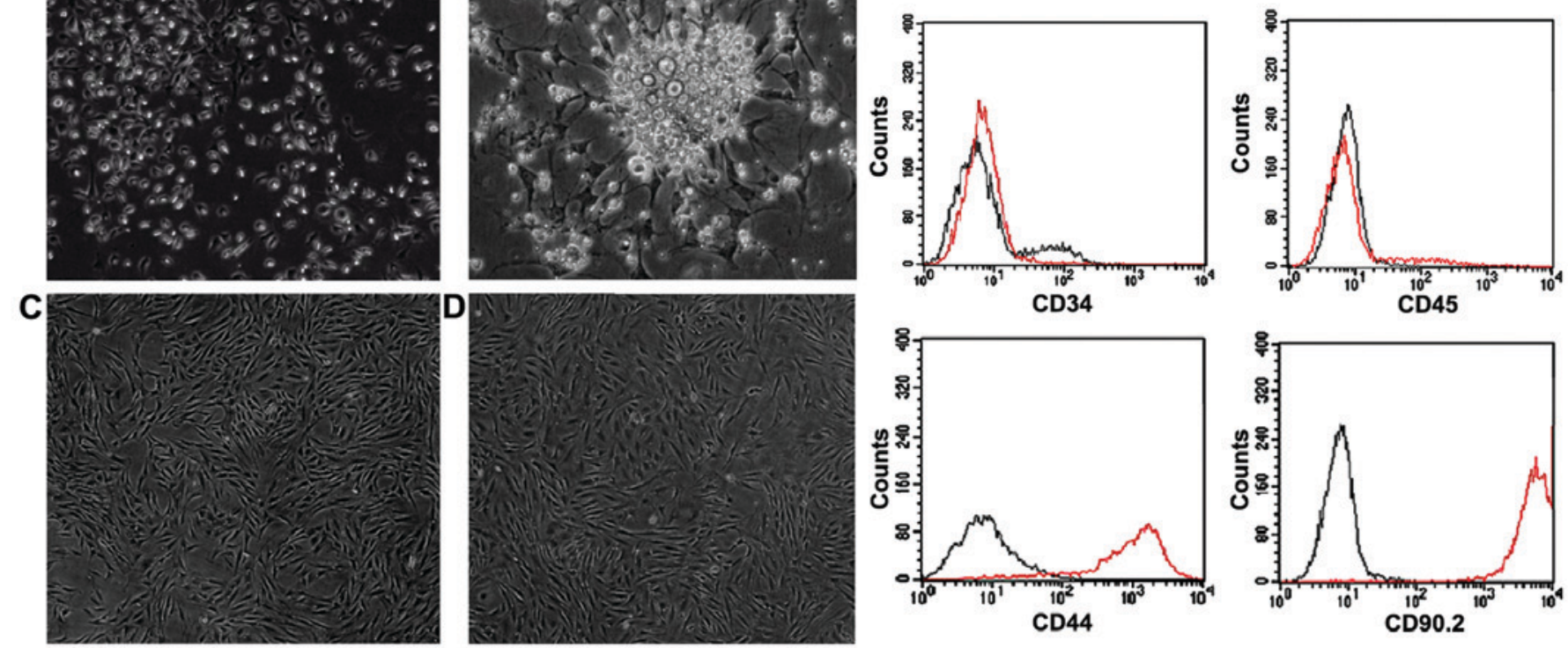

Figure 1. Rat BMSC culture by attachment culture method. (A) Three days after culturing of P0 BMSCs, spindle-like cells scattered (magnification, x100); (B) Five days after culturing of P0 BMSCs, cells grew into colonies (magnification, x200); (C) Fourteen days after culturing of P0 BMSCs, cells reached a density of 90\% (magnification, x100); (D) Five days after culturing of P3 BMSCs, cells showed more uniform morphology and some manifested as wide, fat mature BMSCs (magnification, x100) and (E) Identification of CD molecules of BMSCs with flow cytometry (black as isotype contrast). CD34 (-), CD45 (-), CD44 (+), CD99.2 (+). BMSCs, bone marrow mesenchymal stem cells.

AAATG-3', reverse: 5'-TCCCACTCACATGGTGTCTTCT-3'); collagen X (forward: 5'-GCCCTTTTCCTCTGGCTGAT-3', reverse: 5'-TTGACCAACGTCTGAACAATGG-3'); GAPDH (forward: 5'-TATGACTACCCACGGCAAGT-3', reverse: 5'-ATACTCAGCACCAGCATCACC-3'). We extracted Ct levels when RT-PCR reaction stopped. GAPDH was used as internal control.

Safranin $O$ staining. The cartilage masses were fixed with $4 \%$ paraformaldehyde, paraffin-embedded and then sliced. The slices were differentiated with $1 \%$ ethanol hydrochloride, washed and stained with $0.1 \%$ Safranin O for $5 \mathrm{~min}$. After dehydrated and rinsed by $95 \%$ ethanol, slices were sealed and observed.

Western blot analysis. We lysed BMSCs at 1 week post-induction with RIPA and extracted total proteins. Protein concentration was determined by a protein assay kit. A total of $10 \%$ SDS-PAGE was used to separate protein, and then it was shifted to PVDF membranes (Millipore Corp., Billerica, MA, USA). A total of $5 \%$ fat-free milk was used to block non-specific protein interactions in TBST buffer. The membranes loaded with proteins were incubated at $4^{\circ} \mathrm{C}$ with primary antibody (rabbit anti-rat: Sox 9 and Runx2) and incubated at room temperature with secondary antibody conjugated with horseradish peroxide $(2 \mathrm{~h})$. After washing these membranes in TBST buffer, we developed the membranes using chemiluminescence to detect antibody concentration and took GAPDH as our internal control.

Statistical analysis. SPSS 11.0 (Chicago, IL, USA) was used to analyze our data. Quantitative data are expressed as mean \pm SD. Non-paired t-test was used to analyzed data between groups. A $\mathrm{P}<0.05$ was considered as statistically significant.

\section{Results}

Morphological characteristics and surface marker identification of BMSCs. Small spindle cells, adherently and scattered were observed after BMSCs were changed into medium for 3 days and, dead cells with high grade of refraction were also observed (Fig. 1A). After 4-7 days of culture, the cell volume increased and grew into colonies (Fig. 1B). Fourteen days later, cells showed uniform morphology with spiral pattern and high degree of fusion (Fig. 1C). Five days after passage 3 cells were passaged, cells showed more uniform morphology with a density of $>80 \%$ and some manifested as wide, fat mature BMSCs (Fig. 1D). We cultured and passaged continually and did not observe significant morphology changes. CD molecular phenotype of isolated BMSCs was analyzed with flow cytometry. Results showed that CD34, CD45 (markers of hematopoietic stem cells) were negative and CD44, CD90.2 were positive (Fig. 1E).

Alcian blue staining of BMSCs induced to differentiate to chondroblasts. Alcian blue staining showed that the glycosaminoglycan (GAGs) staining was positive in cell matrix of induced BMSCs and the staining was obviously enhanced with the increased induction time (Fig. 2). It indicated that the isolated BMSCs had the potential of differentiating into cartilage and expressing GAGs which is a cellular phenotype of cartilage.

Collagen II staining of BMSCs induced to differentiate to chondroblasts. We did not detect collagen II staining in BMSCs induced for 1 day but the collagen II staining showed positive with the increased induction time in which staining at 14 days was stronger than that at 7 days (Fig. 3). It indicated that the isolated BMSCs had the potential of differentiating 


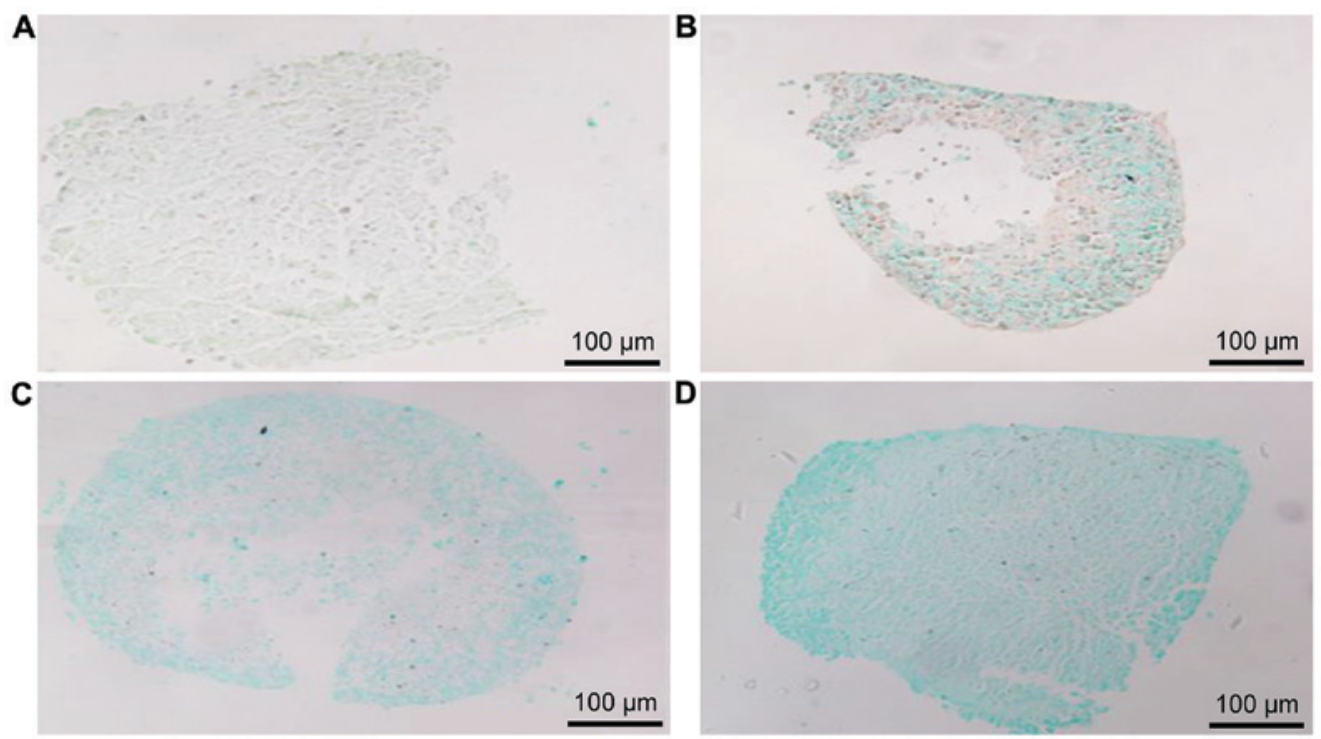

Figure 2. Alcian blue staining of BMSCs at different time points. (A) 1 day after induction, cell mass was fragile and had almost no Alcian blue staining; (B) 3 days after induction, Alcian blue stained at the periphery of cell mass; (C) 7 days after induction, Alcian blue staining was darkened and (D) 14 days after induction, cell mass became dense and staining was more obvious. BMSCs, bone marrow mesenchymal stem cells.
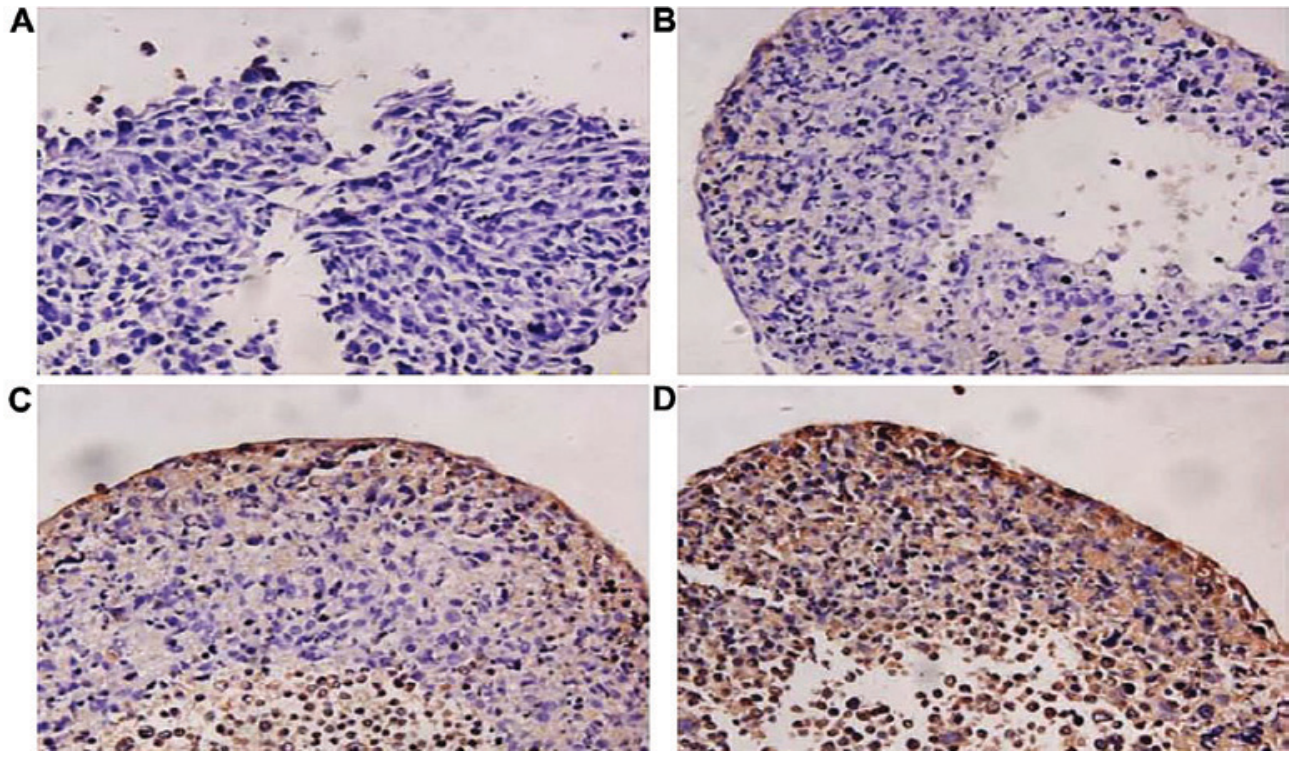

Figure 3. Collagen II staining of BMSCs at different time points. (A) 1 day after induction, cell mass was fragile and collagen II staining was negative. (B) 3 days after induction, collagen II was slightly stained. (C) 7 days after induction, collagen II staining darkened and (D) 14 days after induction, collagen II staining was more obvious. BMSCs, bone marrow mesenchymal stem cells.

into cartilage and expressing collagen II which is a cellular phenotype of cartilage.

Chondroitin sulfate detection by Safranin O staining. Safranin O staining was performed in cartilage micro-groups transfected with miR-127-5p or not to detect chondroitin sulfate. The transfected groups showed vivid red staining while non-transfected groups showed very pale staining. The results showed that miR-127-5p promoted the expression of chondroitin sulfate in cartilage micro-groups induced by BMSCs (Fig. 4).

Cartilage differentiation-related gene expression detection by $R T$-PCR. Cartilage differentiation-related gene expression in
BMSCs induced by induction solution at 7 days were detected by RT-PCR. The results showed that the expression of Sox9, collagen II and aggrecan were higher in the transfected groups than that in control groups with significant difference (Fig. 5A-C). Another finding was that the expression of collagen $\mathrm{X}$ in the transfected groups was lower than that in control groups with significant difference (Fig. 5D).

Results of western blot analysis. Sox 9 and Runx 2 were detected by western blot analysis in BMSCs induced by induction solution at 7 days. Western blot analysis showed that miR-127-5p transfection promoted the expression of Sox9 (Fig. 6A and B) while decreased the expression of Runx2 (Fig. 6C and D) of rat BMSCs with significant difference. 

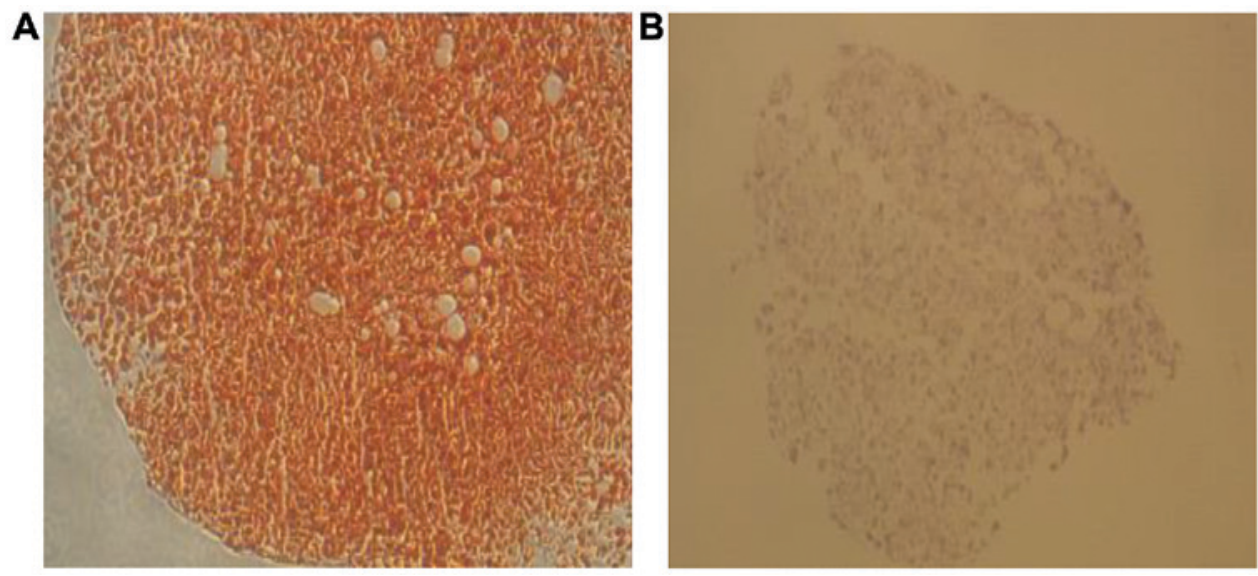

Figure 4. Chondroitin sulfate detection by Safranin O staining (magnification, x100). (A) miR-127-5p transfection group; (B) miRNA control group.

A
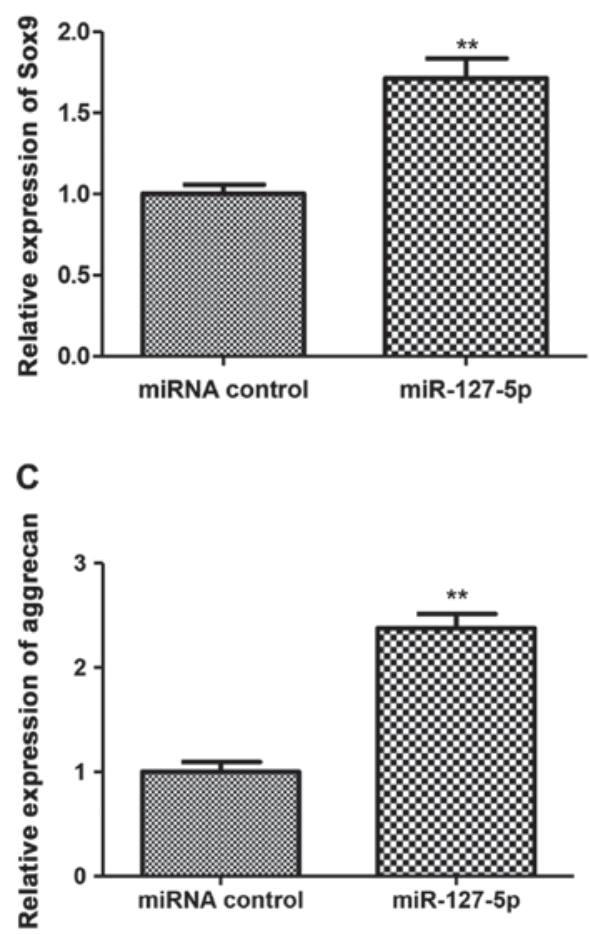

B

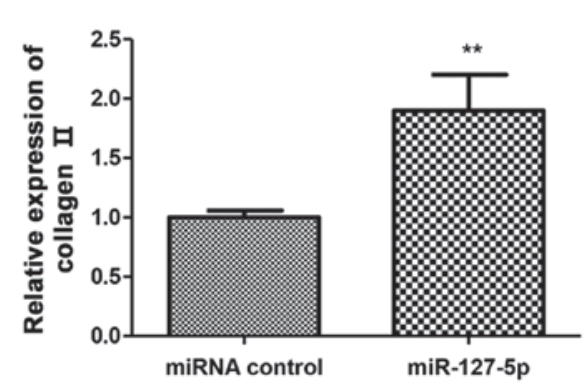

D

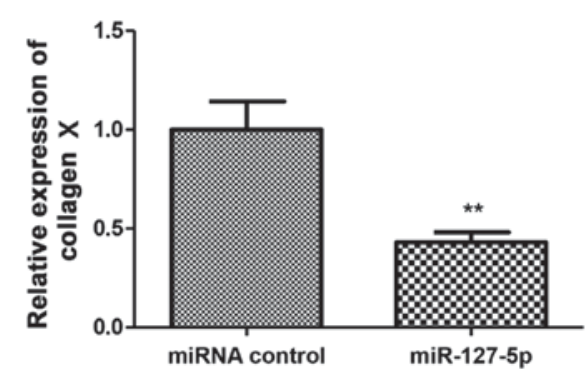

Figure 5. Cartilage differentiation related genes expression detection by RT-PCR. miRNA expressions of (A) Sox9; (B) collagen II and (C) aggrecan were higher in miR-127-5p transfection group than that in miRNA control group, while (D) collagen $\mathrm{X}$ in miR-127-5p transfection group was lower than that in miRNA control group $(* * \mathrm{P}<0.01)$.

\section{Discussion}

miRNA is a new regulator of gene expression. miRNA is of great significance for the proliferation and self-renewal of pluripotent stem cells. It is also very important for the differentiation of mesenchymal stem cells and organ formation. Recently, it was reported that miRNA plays a role in the pathophysiological process of OA $(13,14)$. miR-21 could regulate the expression of GDF-5 and relieve the inflammatory reaction of OA (15). Park et al (12) indicated that miR-127-5p showed obviously downregulation in OA and downregulated MMP-13, inhibited the initiation of OA.

BMSCs, originated from bone marrow pluripotent stem cells, could differentiate into various tissues including bone, cartilage and adipose tissue (16). Research has shown that BMSC differentiation into bone is a highly programmed process and regulated by Runx 2 and finally causes calcium deposition in extracellular matrix. BMSC differentiation into cartilage is regulated by Sox 9 which induces the expression of collagen II and chondroitin sulfate. But one important defect in in vitro induction of BMSC differentiaton into cartilage is that it is difficult to have a stable cartilage phenotype. In the late stage of differentiation, Runx 2 increased and hypertrophic cartilage specific proteins expressed collagen $\mathrm{X}$ decreasing the quality of engineered cartilage. Thus, the engineered cartilage could not compare with hyaline cartilage and form good fusion growth with surrounding cartilage when it is used to repair articular cartilage in vivo. 
A

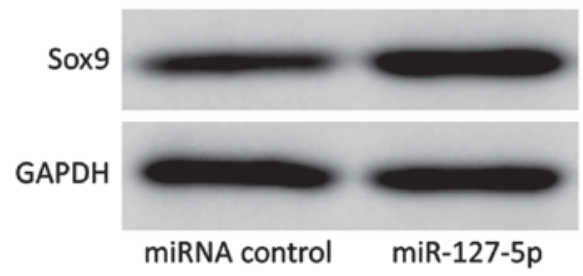

C

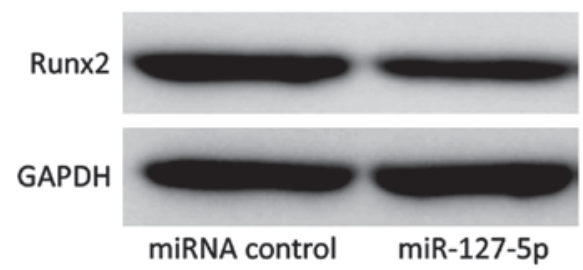

B

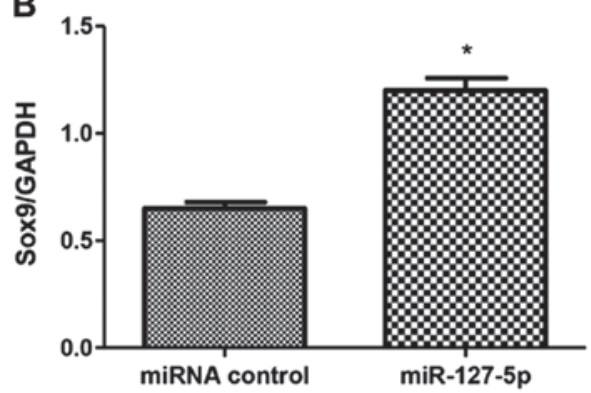

C

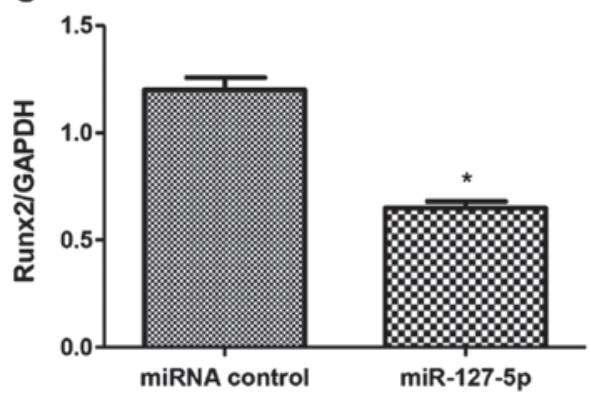

Figure 6. Sox 9 expression was higher in miR-127-5p transfection group than that in miRNA control group. Runx 2 expression was lower in miR-127-5p transfection group than that in miRNA control group. $\mathrm{P}<0.05$.

As a result, downregulating Runx 2 and upregulating Sox 9 may be an effective method to construct engineered cartilage with stable phenotype in the process of BMSC differentiation into cartilage. In the current study, miR-127-5p promoted the expression of chondroitin sulfate in cartilage micro-groups induced by BMSCs. miR-127-5p upregulated the expression of Sox9, collagen II and miR-127-5p while downregulated collagen X. Western blot analysis showed that miR-127-5p could promote the expression of Sox 9 while decreased the expression of Runx2. We inferred that miR-127-5p promotes BMSC differentiation into cartilage and inhibits hypertrophy by regulating core transcripts during bone and cartilage differentiation. Our study revealed that miR-127-5p indeed promoted the BMSC differentiation into cartilage and inhibited its hypertrophy which provided a certain theoretical and experimental basis for constructing engineered cartilage with a stable phenotype through BMSCs. It is of great significance for the treatment of OA.

\section{References}

1. Brittberg M, Lindahl A, Nilsson A, Ohlsson C, Isaksson O and Peterson L: Treatment of deep cartilage defects in the knee with autologous chondrocyte transplantation. N Engl J Med 331: 889-895, 1994.

2. Minas T, Gomoll AH, Solhpour S, Rosenberger R, Probst C and Bryant T: Autologous chondrocyte implantation for joint preservation in patients with early osteoarthritis. Clin Orthop Relat Res 468: 147-157, 2010.

3. de Peppo GM, Svensson S, Lennerås M, Synnergren J, Stenberg J, Strehl R, Hyllner J, Thomsen P and Karlsson C: Human embryonic mesodermal progenitors highly resemble human mesenchymal stem cells and display high potential for tissue engineering applications. Tissue Eng Part A 16: 21612182,2010

4. Varnholt H, Drebber U, Schulze F, Wedemeyer I, Schirmacher P, Dienes HP and Odenthal M: MicroRNA gene expression profile of hepatitis $\mathrm{C}$ virus-associated hepatocellular carcinoma. Hepatology 47: 1223-1232, 2008.
5. Bhaskaran M, Wang Y, Zhang H, Weng T, Baviskar P, Guo Y, Gou D and Liu L: MicroRNA-127 modulates fetal lung development. Physiol Genomics 37: 268-278, 2009.

6. Jiang H, Jin C, Liu J, Hua D, Zhou F, Lou X, Zhao N, Lan Q, Huang Q, Yoon JG, et al: Next generation sequencing analysis of miRNAs: MiR-127-3p inhibits glioblastoma proliferation and activates TGF- $\beta$ signaling by targeting SKI. OMICS 18: 196-206, 2014.

7. Guo LH, Li H, Wang F, Yu J and He JS: The tumor suppressor roles of miR-433 and miR-127 in gastric cancer. Int J Mol Sci 14: 14171-14184, 2013.

8. Zhou J, Lu S, Yang S, Chen H, Shi H, Miao M and Jiao B: MicroRNA-127 post-transcriptionally downregulates Sept7 and suppresses cell growth in hepatocellular carcinoma cells. Cell Physiol Biochem 33: 1537-1546, 2014.

9. Tryndyak VP, Ross SA, Beland FA and Pogribny IP: Downregulation of the microRNAs miR-34a, miR-127, and miR-200b in rat liver during hepatocarcinogenesis induced by a methyl-deficient diet. Mol Carcinog 48: 479-487, 2009.

10. Robertus JL, Harms G, Blokzijl T, Booman M, de Jong D, van Imhoff G, Rosati S, Schuuring E, Kluin P, and van den Berg A: Specific expression of miR-17-5p and miR-127 in testicular and central nervous system diffuse large B-cell lymphoma. Mod Pathol 22: 547-555, 2009.

11. Ying H, Kang Y, Zhang H, Zhao D, Xia J, Lu Z, Wang H, Xu F and Shi L: MiR-127 modulates macrophage polarization and promotes lung inflammation and injury by activating the JNK pathway. J Immunol 194: 1239-1251, 2015.

12. Park SJ, Cheon EJ, Lee MH and Kim HA: MicroRNA-127-5p regulates matrix metalloproteinase 13 expression and interleukin-1 $\beta$-induced catabolic effects in human chondrocytes. Arthritis Rheum 65: 3141-3152, 2013.

13. Nugent M: MicroRNAs: Exploring new horizons in osteoarthritis. Osteoarthritis Cartilage 24: 573-580, 2016.

14. Borgonio Cuadra VM, González-Huerta NC, Romero-Córdoba S, Hidalgo-Miranda A and Miranda-Duarte A: Altered expression of circulating microRNA in plasma of patients with primary osteoarthritis and in silico analysis of their pathways. PLoS One 9: e97690, 2014.

15. Zhang Y, Jia J, Yang S, Liu X, Ye S and Tian H: MicroRNA-21 controls the development of osteoarthritis by targeting GDF-5 in chondrocytes. Exp Mol Med 46: e79, 2014.

16. Panetta NJ, Gupta DM and Longaker MT: Bone regeneration and repair. Curr Stem Cell Res Ther 5: 122-128, 2010. 